$$
\left\{\begin{array}{llll}
a_{1} b_{1} & c_{1} & d_{1} & e_{1} f_{1} \\
a_{2} b_{2} c_{2} d_{2} & e_{2} f_{2} \\
a_{3} b_{3} c_{3} & d_{3} & e_{3} f_{3} \\
a_{4} b_{4} c_{4} d_{4} e_{4} f_{4}
\end{array}\right\} \quad\left\{\begin{array}{llll}
a_{1}-b_{1} & c_{1}-d_{1} & e_{1}-f_{1} \\
a_{2}-b_{2} & c_{2}-d_{2} & e_{2}-f_{2} \\
a_{3}-b_{3} & c_{3}-d_{3} & e_{3}-f_{3} \\
a_{4}-b_{4} & c_{4}-d_{4} & e_{4}-f_{4}
\end{array}\right\}
$$

and is developed into

$$
\begin{gathered}
\Delta \equiv(a)^{2}-\left(\begin{array}{l}
d \\
e
\end{array}\right)^{2}+\left(\begin{array}{l}
d \\
f
\end{array}\right)^{2}+\left(\begin{array}{l}
a \\
e
\end{array}\right)^{2}-\left(\begin{array}{l}
a \\
f
\end{array}\right)^{2}+\left(\begin{array}{l}
b \\
c
\end{array}\right)^{2}+\left(\begin{array}{l}
b \\
f
\end{array}\right)^{2}+ \\
\left(\begin{array}{l}
c \\
e
\end{array}\right)^{2}-\left(\begin{array}{l}
c \\
f
\end{array}\right)^{2} \\
\left.+\left(a_{1} b_{2} e_{3} f_{4}\right)^{2}-\left(a_{1} c_{2} e_{8} f_{4}\right)^{2}+\left(a_{1} d_{2} e_{8} f_{4}\right)^{2}+b_{1} c_{2} e_{3} f_{4}\right)^{2}- \\
\left(b_{1} d_{2} e_{8} f_{4}\right)^{2}+\left(c_{1} d_{2} e_{3} f_{4}\right)^{2}
\end{gathered}
$$

'The first row is the same as the coefficient of $(a)^{2}$ in $\Phi$, and from determinants,

$$
(a)_{1}^{2}\left(c_{1} d_{2} e_{3} f_{4}\right)^{2}=\left[\left(\begin{array}{l}
a \\
e
\end{array}\right)\left(\begin{array}{l}
b \\
f
\end{array}\right)-\left(\begin{array}{l}
a \\
f
\end{array}\right)\left(\begin{array}{l}
b \\
e
\end{array}\right)\right]^{2} \text {, and similarly for }
$$

the other terms, then $\Delta \equiv(a)^{2} \Phi$. The two lines common to four linear complexes are real, coincident or imaginary according as the combinant of the complexes is positive, zero or negative. This criterion may be used to find the reality of the lines cutting four given ones, by making the principal diagonal of $\Delta$ vanish, and for $A_{i k}$, the polar of the lines $i, k$.

CoRnell UNIVERSITY, January 8, 1897.

\title{
THE CUBIO RESOLVENT OF A BINARY QUARTIC DERIVED BY INVARIANT DEFINITION AND PROCESS.
}

\author{
BY PROFESSOR H. s. WHITE.
}

(Read before the Conference at Chicago, January 1, 1897.)

In the usual discussion of a binary quartic the cubic resolvent first arises as an auxiliary in factoring the quantic, or what is the same thing, in reducing it to a determinate normal form. The coefficients of this cubic, when found, prove to be rational invariants of the original quartic. Such a fact appears as a surprise, since the invariant character of the roots of the cubic is rarely made prominent at 
the beginning of the inquiry. As a supplement to such discussions, the following derivation of the resolvent cubic has the merit of employing invariant definitions and processes throughout. It might properly precede the application of this cubic to the reduction or solution of the quartic.

As a preliminary step, consider two quadrics : $P=p_{x}^{2}$ and $Q=q_{x}^{2}$, together with their first transvectant: $A=a_{x}^{2}=$ $(P, Q)=(p q) p_{x} q_{x^{\circ}}$ The form $A$ has then this property (completely defining it) that its second transvectant with either of the quadrics $P$ and $Q$ vanishes identically. Each of these three quadrics, equated to zero, is represented geometrically by two points of a line, and the two points $A$ divide harmonically both the pair $P$ and the pair $Q$. These well known relations are proved by the identities:

$$
\begin{aligned}
& (P, A)_{2}=\left(p^{\prime} \alpha\right)^{2}=(p q)\left(p^{\prime} p\right)\left(p^{\prime} q\right)=\frac{1}{2}\left(p^{\prime} p\right)^{2}(q q) \equiv 0, \\
& (Q, A)_{2}=\left(q^{\prime} \alpha\right)^{2}=(p q)\left(q^{\prime} p\right)\left(q^{\prime} q\right)=\frac{1}{2}\left(q^{\prime} q\right)^{2}(p p) \equiv 0 .
\end{aligned}
$$

The form $\alpha_{x}^{2}$ satisfies these two invariant equations rational in the coefficient of $p_{x}^{2}$ or $q_{x}^{2}$. But further, if we consider the quartic composed of the quadric factors $p_{x}^{2}$ and $q_{x}^{2}$ :

$$
f=a_{x}^{4}=p_{x}^{2} \cdot q_{x}^{2},
$$

the form $\alpha_{x}^{2}$ satisfies a peculiar invariant equation which is rational in the coefficients of this quartic $f$ and one adjoined quantity $\lambda$. The second transvectant, namely, of quartic and quadric is a constant multiple of the quadric $\alpha_{x}^{2}$ itself. The proof is as follows :

$$
\begin{aligned}
& 4(f, A)_{2}=4(\alpha \alpha)^{2} a_{x}^{2}= \\
& =(p q)\left\{\left(p p^{\prime}\right)\left(q p^{\prime}\right) q_{x}^{2 \prime}+\left(p q^{\prime}\right)\left(q q^{\prime}\right) p_{x}^{2 \prime}+\right. \\
& \left.\left(p p^{\prime}\right)\left(q q^{\prime}\right) p_{x}^{\prime} q_{x}^{\prime}+\left(p q^{\prime}\right)\left(q p^{\prime}\right) p_{x}^{\prime} q_{x}^{\prime}\right\} .
\end{aligned}
$$

Of these four terms the first two vanish identically, as was seen above; the third reduces thus:

$$
\left(p p^{\prime}\right)\left(q q^{\prime}\right)(p q) p_{x}^{\prime} q_{x}^{\prime}=\frac{1}{2}\left(p p^{\prime}\right)^{2} \cdot\left(q q^{\prime}\right) q_{x} q_{x}^{\prime} \equiv 0 ;
$$

the fourth becomes, by similar reduction,

$$
-\frac{1}{2}(p q)^{2} \cdot\left(p^{\prime} q^{\prime}\right) p_{x}^{\prime} q_{x}^{\prime}=-\frac{1}{2}(p q)^{2} \cdot \alpha_{x}^{2},
$$

the quadric $\alpha_{x}^{2}$ multiplied by a constant $-\frac{1}{2}(p q)^{2}$.

Here we may begin de novo and inquire : having given a quartic $\alpha_{x}^{4}$, how many quadrics $\alpha_{x}^{2}$ can be found to satisfy identically a transvectant reproductive equation 


$$
(\alpha \alpha)^{2} a_{x}^{2} \equiv \lambda \cdot \alpha_{x}^{2},
$$

and what is the equation determining $\lambda$ ? This question is invariant in form, showing that the constants $\lambda$ and the corresponding quadrics $\alpha_{x}^{2}$, if determinate, must be invariants and covariants of the quartic $a_{x}^{4}$.

The characteristic equation is quadric, and yields three independent linear equations for the $a$ 's. Hilbert has eliminated from these the co-efficients of $\alpha_{x}^{2}$ and reduced the resulting cubic determinant in $\lambda_{.} *$ But the same end may be accomplished without leaving the domain of invariant equations, by using an identity elegantly proved by Clebsch. Repeated use of the defining equations gives the series of relations all equivalent to the first:

$$
\begin{aligned}
(a \alpha)^{2} a_{x}^{2} & =\lambda \cdot \alpha_{x}^{2} \\
(a \alpha)^{2}(a b)^{2} b_{x}^{2} & =\lambda^{2} \cdot \alpha_{x}^{2} \\
(a \alpha)^{2}(a b)^{2}(b c)^{2} c_{x}^{2} & =\lambda^{3} \cdot \alpha_{x}^{2}, \text { etc. }
\end{aligned}
$$

Using Clebsch's notation, call $P$ the operation of deriving $(a \alpha)^{2} a_{x}^{2}$ from $\alpha_{x}^{2}-$

$$
P\left(\alpha_{x}^{2}\right)=(\alpha \alpha)^{2} a_{x}^{2}
$$

then his identical relation is

$$
P^{3}\left(\alpha_{x}^{2}\right) \equiv \frac{i}{2} \cdot P\left(\alpha_{x}^{2}\right)+\frac{j}{3} \cdot \alpha_{x}^{2} \dagger
$$

$i$ and $j$ denoting the fundamental invariants of the quartic :

$$
i=(a b)^{4}, j=(a b)^{2}(b c)^{2}(c a)^{2} .
$$

By virtue of the above relations we have, however :

$$
\left(P^{3}-\frac{i}{2} P-\frac{j}{3} \cdot\right)\left(\alpha_{x}^{2}\right)=\left(\lambda^{3}-\frac{i}{2} \cdot \lambda-\frac{j}{3}\right) \cdot \alpha_{x}^{2}=0 .
$$

Hence, if $\alpha_{x}^{2}$ be a determinate form, we must have $\lambda$ a root of the equation

$$
\lambda^{3}-\frac{i}{2} \lambda-\frac{j}{3}=0
$$

the reducing cubic.

Looking back to the point where $\lambda$ was first introduced, we see it denoting an irrational invariant $-\frac{1}{8}(p q)^{2}$ of the quartic. There are, in general, as there must be in ac-

* Math. Annalen, Bd., 28, p. 433.

† Binäre Formen, p. 219. 
cordance with this fact, three possible values of $\lambda$, and to each a corresponding quadric linearly determined.

A known invariant identity has yielded readily the determining equation for the irrational invariant $\lambda$. I intend at another time to show that the reverse process is legitimate, direct and fruitful.

NORTHWESTERN UNIVERSITY, January, 1897.

\section{TWO BOOKS ON ELEMENTARY GEOMETRY.}

Elements of Geometry. By Andrew W. Phillips, Ph.D., and Irving Fisher, PH.D., Professors in Yale University. New York, Harper and Bros. 1896.540 pp. $\quad \$ 1.75$.

Elementary Solid Geometry and Mensuration. By Henry DaLlas Thompson, D.Sc., Pн.D., Professor of Mathematics in Princeton University. New York and London, The Macmillan Company. 1896. vii+199 pp. $\$ 1.25$.

Among the multitude of books on elementary geometry brought out within the last few years, two that have appeared recently rise above the general level and call for notice.

The larger and more ambitious of these- "Elements of Geometry," by Phillips and Fisher, a work of over 500 pages-covers the ground of the ordinary school text-books on plane and solid geometry, and makes the rough places smooth before the pupil with all the modern equipments in the way of realistic diagrams and conspicuous and varied types. We are tempted to wonder whether by thus diminishing the necessity for the exercise of the imagination and the attention, we may not lose some of the qualities which render geometry invaluable as a mental discipline. A noteworthy innovation is the final chapter, headed an "Introduction to Modern Geometry," which gives a brief accountso brief as to be scarcely more than a summary-of inversion, linkages, stereographic projection, poles and polars, the nine-points circle, perspective, duality, involution, antiparallels and the axioms of plane, spherical and pseudospherical geometries.

The chief novelties to be noted in the first part of the book are the early introduction of the idea of symmetry and the use of rotation about a point to prove theorems, relating to parallel lines. The note on p. 23, designed ap- 